\title{
EFECTO DE LA ACUMULACIÓN DE FACTORES DE RIESGO EN EL RIESGO DE CARCINOMA DE
} CÉLULAS ESCAMOSAS BUCAL

\author{
EFEITO DO ACÚMULO DE FATORES DE RISCO SOBRE O RISCO DE CARCINOMA EPIDERMÓIDE ORAL \\ EFFECT OF THE ACCUMULATION OF RISK FACTORS ON THE RISK OF ORAL SQUAMOUS CELL CARCINOMA \\ Eduardo David PIEMONTE ${ }^{1}$, Jerónimo Pablo LAZOS ${ }^{2}$, Paola BELARDINELLI², Graciela del Valle CASTILLO4, Angel Daniel
}

\author{
TALAVERA ${ }^{2}$, Dante Gustavo SECCHI ${ }^{2}$, Héctor Eduardo LANFRANCHI TIZEIRA ${ }^{7^{\dagger}}$, Mabel Noemí BRUNOTTO ${ }^{8}$
}

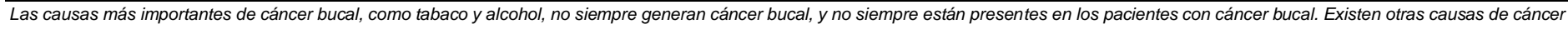

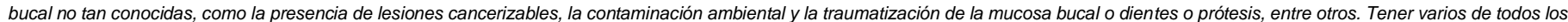

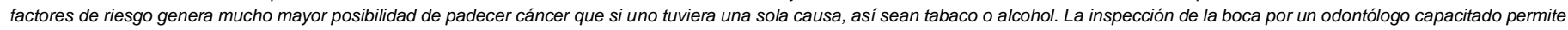
detectar a los pacientes que presentan mayor riesgo de cáncer bucal.

Conceptos claves:

A) ¿Qué se sabe sobre el tema?

Los factores de riesgo de carcinoma de células escamosas, incluso los más conocidos como tabaco y alcohol, no pueden explicar la totalidad de estos cánceres bucales.

Existen otros factores de riesgo de cáncer bucal menos explorados en la investigación, que son prevalentes en Córdoba

Poco se conoce sobre la sumatoria de efectos de los diferentes factores de riesgo de carcinoma de células escamosas, a nivel individual

B) ¿Qué aporta este trabajo?

Existen factores de riesgo de carcinoma de células escamosas que podrían generar mayor riesgo que tabaco y alcohol en la población de Córdoba.

La presencia de Desórdenes Potencialmente Malignos, infección por VPH e Irritación Mecánica Crónica en la mucosa bucal, y el antecedente de haber tomado agua de pozo en áreas de contaminación arsenical podrían los factores de riesgo más importantes que tabaco y alcohol para el carcinoma de células escamosas en la población cordobesa.

La acumulación de factores de riesgo de carcinoma de células escamosas sería el factor de riesgo más importante para el carcinoma de células escamosas, y constituye una causa necesaria para el desarrollo de dicho cáncer.

1 Profesor Adjunto, Cátedra de Estomatología "A", Facultad de

Odontología, Universidad Nacional de Córdoba.

2 Profesor Asistente, Cátedra de Estomatología "A", Facultad de Odontología, Universidad Nacional de Córdoba.

3 Profesor Asistente, Cátedra de Química Biológica "B", Facultad de Odontología, Universidad Nacional de Córdoba

4 ex Profesor Titular, Cátedra de Clínica Estomatológica, Facultad de Odontología, Universidad de Buenos Aires, ORCID 0000-00024596-2671

5 Profesora Titular, Cátedra de Biología Celular "A", Facultad de Odontología, Universidad Nacional de córdoba, ORCID 00000001-8010-1079

Recibido: 2020-12-11 Aceptado: 2021-03-21

DOI: http://dx.doi.org/10.31053/1853.0605.v78.n2.31247 (c) (i) (8)

CUniversidad Nacional de Córdoba

\section{Resumen:}

Introducción: los factores de riesgo de carcinoma de células escamosas bucal son causas no necesarias y no suficientes, incluso los más aceptados, como tabaco y alcohol. Poco se conoce sobre el efecto acumulativo de todos los factores de riesgo de cáncer bucal. El objetivo de este trabajo fue analizar si la sumatoria de factores de riesgo de cáncer bucal se asocia con un aumento de su riesgo.

Métodos: se realizó un estudio de casos y controles para determinar los factores de riesgo de cáncer bucal en la población bajo estudio, para luego correlacionar el número de factores de riesgo en cada individuo, con el riesgo de cáncer bucal.

Resultados: la acumulación de factores de riesgo de cáncer bucal, analizada mediante diferentes estrategias, estuvo siempre asociada con un aumento significativo del riesgo de cáncer bucal. Tener 3 o más factores de riesgo estuvo asociado con mayor riesgo de cáncer bucal (OR 110, p<0,0001)

Conclusión: el principal factor de riesgo de cáncer bucal es la acumulación de sus factores de riesgo. La acumulación de factores de riesgo podría considerarse una causa necesaria para el desarrollo de cáncer bucal.

Palabras clave: neoplasias de la boca; carcinoma de células escamosas; factores de riesgo; causalidad

\section{Abstract:}

Introduction: the risk factors of oral squamous cell carcinoma are not necessary and not sufficient causes, even the most accepted ones, such as tobacco and alcohol. Little is known about the cumulative effect of all risk factors for oral cancer. The objective of this work was to analyze whether the sum of oral cancer risk factors is associated with an increase in its risk.

Methods: a case-control study was carried out to determine the risk factors for oral cancer in the population under study, and then correlate the number of risk factors in each individual with the risk of oral cancer. Results: the accumulation of oral cancer risk factors, analyzed using different strategies, was always associated with a significant increase in the risk of oral cancer. Having 3 or more risk factors was associated with an increased risk of oral cancer (OR 110, $p<0.0001$ )

Conclusion: the main risk factor for oral cancer is the accumulation of its risk factors. The accumulation of risk factors could be considered a necessary cause for the development of oral cancer.

Key words: mouth neoplasms; squamous cell carcinoma; risk factors; causality

\section{Resumo}

Introdução: os fatores causais do carcinoma epidermóide oral não são causas necessárias e nem suficientes, mesmo os mais aceitos, como o fumo e o álcool. Pouco se sabe sobre o efeito cumulativo de todos os fatores de risco para câncer oral. O objetivo deste trabalho foi analisar se a soma dos fatores de risco do câncer bucal está associada a um aumento do seu risco.

Métodos: foi realizado um estudo caso-controle para determinar os fatores de risco para câncer bucal na população em estudo e, em seguida, correlacionar o número de fatores de risco em cada indivíduo com o risco de câncer bucal.

Resultados: o acúmulo de fatores de risco para câncer bucal, analisados por meio de diferentes estratégias, sempre esteve associado a um aumento significativo no risco de câncer bucal. Ter 3 ou mais fatores de risco foi associado a um risco aumentado de câncer oral (OR 110, $p<0,0001$ )

Conclusão: o principal fator de risco para o câncer bucal é o acúmulo de seus fatores de risco. O acúmulo de fatores de risco pode ser considerado uma causa necessária para o desenvolvimento do câncer bucal.

Palabras chave: neoplasias bucais; carcinoma de células escamosas; fatores de risco; causalidade 


\section{Introducción}

La carcinogénesis es un proceso multifactorial y multietápico. El concepto actual de multicausalidad implica que una enfermedad se produce generalmente por un conjunto de causas no necesarias y no suficientes. Que sea no suficiente significa que cualquier causa o factor necesita de al menos otro factor para producir la enfermedad. Y que no sea necesaria, significa que la presencia de un factor no está siempre asociada a la aparición de una determinada enfermedad ${ }^{(1)}$.

El carcinoma de células escamosas bucal (CCEB) cumple estas reglas de multifactorialidad ${ }^{(2)}$. Si bien el tabaco y el alcohol han sido reconocidos como los factores de riesgo más importantes, varios hechos demuestran que los factores causales de CCEB son no necesarios y no suficientes. El CCEB se produce en pacientes no fumadores ni bebedores, sino asociado a otras causas, lo que demuestra que ni siquiera estos factores mayores son causas necesarias. Por otra parte, el consumo de tabaco y alcohol, incluso en grandes cantidades, por individuos que nunca desarrollan CCEB, demuestra que tampoco son suficientes ${ }^{(3)}$. Por ejemplo, de los pacientes fumadores pesados que hay en toda la población, sólo un porcentaje menor al $5 \%$ desarrolla $\mathrm{CCEB}^{(4)}$. Este hecho podría ser explicado por la interacción con otros factores de riesgo.

En un proceso de carcinogénesis siempre es necesario que exista al menos un factor iniciador y un factor promotor para que la carcinogénesis pueda completarse ${ }^{(1)}$. Todos ellos pueden interactuar no sólo para que se produzca una enfermedad, sino también para que lo haga con mayor frecuencia y/o gravedad. Para estudiar estos procesos multicausales es necesario analizar las interacciones epidemiológicas y biológicas entre los distintos factores. Sin embargo, en el área de cáncer bucal el estudio de la causalidad estuvo generalmente centrado en la interacción de tabaco y alcohol, y en menor medida en otros factores como betel, HPV y dieta ${ }^{(5)}$. Existen otros factores de CCEB menos reconocidos o controversiales, algunos de ellos muy prevalentes en la población mundial, como candidiasis, enfermedad periodontal e irritación mecánica crónica (IMC) de la mucosa bucal ${ }^{(6)}$. Además, en Argentina existen factores de riesgo propios, como la contaminación geológica con arsénico del agua de consumo humano, que afecta predominantemente a poblaciones rurales o de pequeños pueblos, por encima no sólo de los valores que establece la OMS, sino también sobre las normativas nacionales ${ }^{(7)}$. El consumo de mate, altamente prevalente en nuestro país y otros países limítrofes, ha sido propuesto también como un factor de riesgo para CCEB, principalmente debido a la alta temperatura de la infusión ${ }^{(8)}$.

Si bien de algunos de ellos se han realizado estudios sobre interacción con otros factores, poco se sabe sobre la interacción entre todos ellos a nivel epidemiológico y biológico. La presencia de múltiples factores de riesgo de CCEB en un mismo individuo, podría aumentar su riesgo de CCEB.

Por todo esto, los objetivos de este trabajo fueron determinar los factores de riesgo de CCEB en la población bajo estudio y analizar el riesgo de CCEB según el número de factores presentes en cada individuo.

\section{Metodología}

Se realizó un estudio de casos y controles en la Facultad de Odontología de la Universidad Nacional de Córdoba, Argentina, desde 2009 a 2103. El estudio fue aprobado por el Consejo de Evaluación Ética de la Investigación en Salud dependiente del Ministerio de Salud de la Provincia de Córdoba ( $\left.N^{\circ} 1378\right)$. Todos los pacientes firmaron el consentimiento informado.

El criterio de inclusión para el grupo de estudio fue el diagnóstico histopatológico de carcinoma de células escamosas en mucosa bucal (ICD-10 C00-C06). Los criterios de exclusión fueron el diagnóstico de cáncer originado en la semimucosa labial y el tratamiento previo de cáncer de cabeza y cuello con cirugía y/o radioterapia, o estar recibiendo quimioterapia. El grupo de control fue constituido por pacientes sin CCEB que concurrieron buscando tratamiento odontológico general. Se excluyeron del grupo control pacientes cuyo motivo principal de consulta hubiera sido un desorden potencialmente maligno (DPM) o una lesión bucal asociada a IMC.

\section{Recolección de datos}

Los pacientes fueron entrevistados y examinados por odontólogos calibrados en el diagnóstico de enfermedades de la mucosa bucal. Las siguientes variables fueron registradas mediante anamnesis:

Edad: $>45$ años $y \leq 45$ años

Sexo: femenino o masculino

Tabaco: la exposición acumulada a tabaco se obtuvo multiplicando el promedio diario por los años de consumo, por 365, expresándose el resultado en número de cigarrillos. Se consideró fumador a quien que fumó ocasional o regularmente por más de un año un promedio de un cigarrillo diario.

Tabaquismo pasivo: se registró en años de exposición.

Alcohol: se estimó la exposición acumulada a bebidas alcohólicas multiplicando los litros semanales consumidos de cada tipo de bebida, por los años de consumo, por 52. Los litros obtenidos se multiplicaron por 8 y por la concentración de alcohol para cada tipo de bebida (cerveza $4,5 \%$ vino $13 \%$, y bebidas destiladas o licores $30 \%$ ). Los subtotales de cada bebida fueron sumados para obtener una exposición total expresada en gramos de alcohol. Se consideró bebedor a quien bebió alcohol regularmente por más de un año.

Mate: promedio de consumo diario expresado en litros.

Arsénico en agua de consumo humano: consumo de agua de pozo en zona arsenicales, durante 10 años o más.

Carcinógenos ambientales: exposición laboral o ambiental a sustancias potencialmente carcinogénicas, excluyendo arsénico.

Historia familiar de cáncer: diagnóstico de cáncer en parientes de primer o segundo grado.

Dieta Protectora: consumo semanal de alimentos con efecto protector de cáncer bucal: vegetales frescos o cocidos (no fritos ni asados ni en guisos con carne), frutas y lácteos. 
Las variables siguientes fueron registradas mediante inspección clínica: Dientes perdidos: número de dientes extraídos o con extracción indicada. Los criterios para la indicación de extracción fueron caries subgingival a nivel de cresta ósea, enfermedad periodontal con movilidad grado III, y malposición severa no solucionable con ortodoncia.

Enfermedad periodontal: se registró pérdida de inserción clínica, incluyendo el mayor valor encontrado al sondaje con sonda periodontal. Si el paciente hubiera perdido algún diente por enfermedad periodontal, se le asignó el mayor valor registrado entre los pacientes.

Irritación mecánica crónica: se consideró presente sobre mucosa previamente sana o sobre patologías preexistentes, cuando se encontró cualquiera de los siguientes criterios $^{(9)}$ :

- Lesión clínica compatible con origen traumático, de un mes o más de evolución, con contacto estático, o por movimientos funcionales o parafuncionales (deglución disfuncional, interposición lingual, masticación unilateral, succión, mordisqueamiento, estabilización de prótesis con la lengua o labios, entre otros) con cualquier agente potencialmente traumatizante de origen dentario o protético, el cual debía existir antes de la lesión, o del agravamiento de una lesión preexistente.

-Historia de úlcera bucal crónica o recurrente, durante más de un mes, en el sitio de posterior aparición del CCEB, en relación con factores de IMC dentarios, protéticos y/o funcionales.

-CCEB con contacto estático, funcional o parafuncional con cualquier factor dentario o protético potencialmente traumatizante, siempre que el supuesto factor hubiera existido en boca con anterioridad a la percepción del CCEB por parte del paciente. Este dato fue obtenido mediante anamnesis y/o inspección bucal.

Desórdenes potencialmente malignos: acorde a los criterios de la $\mathrm{OMS}^{(10)}$.

Candidiasis crónica: lesión clínica compatible con candidiasis crónica acompañada de identificación de Candida por microscopía directa o cultivo de muestras obtenidas mediante citología exfoliativa.

VPH: la presencia de VPH fue considerada posible cuando existieron lesiones clínicas, debido a que su presencia en mucosa sana no es indicadora de enfermedad, sino probablemente de una infección transitoria. La presencia de VPH fue considerada positiva en lesiones clínicas con PCR para VPH positiva.

\section{Análisis estadístico}

Las variables cualitativas fueron categorizadas en forma binaria. Las variables cuantitativas fueron categorizadas de forma binaria según la mediana del grupo control, excepto edad y tabaco (Tabla 1).

Las variables DPM, IMC, candidiasis crónica, pérdida dentaria y enfermedad periodontal fueron registradas en 20 pacientes por dos operadores para analizar la concordancia interobservador mediante test tau-b de Kendall y eliminar del análisis estadístico a variables con concordancia menor a 0,6.

El análisis bivariado se realizó utilizando $X^{2}$ para excluir variables no significativas. El análisis multivariado se realizó en dos etapas. En la primera se realizó regresión logística ajustada por edad, género, tabaco y alcohol, para cada variable. Aquellas que resultaron significativas fueron incluidas en el modelo final de regresión logística.

Para cada paciente se sumó un punto por cada variable significativa que tuviera presente, se categorizó a los pacientes según el número de factores presentes (de 0 a 8), y luego en forma binaria según mayor o menor número de factores. Para cada categorización se analizó la relación con riesgo de cáncer bucal utilizando $X^{2}$. Se consideró significativo un valor de $p<0,05$. Se utilizó software Infostat, versión 2020 (www.infostat.com.ar).

\section{Resultados}

Fueron incluidos en el presente estudio 53 pacientes ( 29 hombres y 24 mujeres) del grupo de estudio y 100 pacientes (36 hombres y 64 mujeres) del grupo control. La edad de los pacientes incluidos tuvo una media de 52,4 años, con un rango de 19 a 88 años. En el grupo de estudio la edad media fue de 63 años, con un rango entre 23 y 88 años; mientras que en el grupo control la media fue de 46,7 años con un rango entre 19 y 74 años. El número de casos de CCEB y su respectivo porcentaje, según ubicación de las lesiones fue la siguiente: lengua (salvo 1 en cara ventral, todas en borde) 27 (51\%); encía (incluyendo reborde desdentado) 8 (15\%); piso de boca $5(9 \%)$; mucosa yugal 5 $(9 \%)$; paladar duro $3(6 \%)$; pilares anteriores $2(4 \%)$; fondo de surco vestibular 2 (4\%); y mucosa labial $1(2 \%)$.

Todas las variables de registro mediante inspección clínica presentaron un coeficiente de concordancia interobservador de 0,6 o superior, por lo que ninguna fue excluida del estudio por este motivo. En el grupo de estudio hubo 16 pacientes desdentados totales y por ese motivo se excluyó a la enfermedad periodontal del modelo estadístico. Con los pacientes en los cuales se pudo registrar esta variable $(n=135$ : grupo de estudio 37; grupo control 98), el test de X2 mostró un mayor riesgo de CCEB para aquellos individuos con más de $5 \mathrm{~mm}$ de pérdida de inserción (OR de 3,6 IC 95\% 1,57-8,24, p=0,0018).

El análisis bivariado indicó que las variables candidiasis crónica, tabaquismo pasivo, exposición a carcinógenos laborales y ambientales, dieta e historia familiar de cáncer no presentaron aumento de riesgo significativo de CCEB, por lo cual fueron excluidos del estudio multivariado (Tabla 1). La primera etapa del análisis multivariado permitió excluir a la variable dientes perdidos por resultar no significativa (Tabla 2). Las variables restantes conformaron la segunda etapa del modelo multivariado, el cual mostró que, a excepción de género y consumo de alcohol, todas las variables estuvieron asociadas con un mayor riesgo de CCEB, estadísticamente significativo (Tabla 3). Sin embargo, a pesar de no ser significativa en esta etapa, la variable consumo de alcohol fue mantenida en el modelo de análisis final debido a que existen estudios de alto nivel de evidencia científica que indican que es un factor de riesgo de CCEB.

En cuanto al número de factores presentes, el rango fue desde 0 a 7 factores en un mismo paciente, con un promedio del total en la población de 2,72 factores. Sin embargo existió una diferencia estadísticamente significativa en el promedio de factores según cada 
grupo, con un promedio de 4,32 para el grupo de CCEB contra un promedio de 1,87 del grupo control ( $p<0,0001$, test $t$ de Student). La distribución de los pacientes según la cantidad de factores presentes se observa en figura 1. Los pacientes con 0 a 2 factores prácticamente no presentaron CCEB, ya que con 2 factores sólo hubo un caso de CCEB, y con 1 o ninguno factor no hubo ningún caso de CCEB. Inversamente, entre los pacientes con 5 o más factores sólo hubo un caso de control entre 22 pacientes con CCEB. $Y$ en los pacientes con 3 y 4 factores hubo una cantidad similar de casos y de controles en números reales, pero porcentualmente el número de casos casi duplicó al de controles. Debido a esta distribución se categorizó adicionalmente en 3 categorías. Mediante $X^{2}$ se observó que cualquiera sea la forma en que se categorice el número de factores presentes en cada paciente, el tener más factores de riesgo estuvo estadísticamente asociado de manera significativa con un mayor riesgo de CCEB (Tabla 4). Para testear la confiabilidad de la categorización según el número de factores de 0 a 8 , se realizó una curva ROC cuyo resultado indicó un área bajo curva de 0,9211 (E.E. 0,0268; $p$-<0,0001).

\begin{tabular}{|c|c|c|c|c|c|c|c|c|}
\hline \multirow{2}{*}{ Variable } & \multirow{2}{*}{ Categoría } & \multirow{2}{*}{ Control } & \multirow{2}{*}{ CCEB } & \multirow{2}{*}{$x^{2}$} & \multirow{2}{*}{ OR } & \multicolumn{2}{|c|}{$\mathrm{IC} 95 \%$} & \multirow{2}{*}{$p$} \\
\hline & & & & & & $\mathrm{LI}$ & LS & \\
\hline \multirow[t]{2}{*}{ Desórdenes potencialmente malignos } & $\mathrm{No}^{*}$ & 97 & 30 & \multirow{2}{*}{40.0} & \multirow{2}{*}{24.7} & \multirow{2}{*}{7.5} & \multirow{2}{*}{81.8} & \multirow{2}{*}{0.0001} \\
\hline & Sí & 3 & 23 & & & & & \\
\hline \multirow{2}{*}{ VPH } & $\mathrm{No}^{*}$ & 97 & 37 & \multirow{2}{*}{23.5} & \multirow{2}{*}{13.9} & \multirow{2}{*}{4.1} & \multirow{2}{*}{47.0} & \multirow{2}{*}{0.0001} \\
\hline & Sí & 3 & 16 & & & & & \\
\hline \multirow{2}{*}{ Consumo de agua de pozo en zona arsenical $^{\circ}$} & $<10^{*}$ & 96 & 38 & \multirow{2}{*}{18.8} & \multirow{2}{*}{9.4} & \multirow{2}{*}{2.9} & \multirow{2}{*}{30.3} & \multirow{2}{*}{0.0001} \\
\hline & $100+$ & 4 & 15 & & & & & \\
\hline \multirow{2}{*}{$\mathrm{Edad}^{\circ}$} & $450-$ & 47 & 6 & \multirow{2}{*}{19.4} & & & & \\
\hline & $>45$ & 53 & 47 & & 0.0 & 2.1 & 11.1 & $<0.000$ \\
\hline Irritación mecánica crónica & $\mathrm{No}^{*}$ & 64 & 15 & & & & & \\
\hline & Sí & 36 & 38 & & & 2.2 & 0.5 & 0.0001 \\
\hline Consumo acumulado de alcohol & $<72793 \mathrm{gr}^{*}$ & 81 & 25 & 168 & 14 & 01 & 01 & מחח \\
\hline & $=0>72793 \mathrm{gr}$ & 19 & 28 & & 4.4 & 2.1 & 0.1 & $<0.0001$ \\
\hline Mate & $<1$ lt/día * & 50 & 14 & & & & & \\
\hline 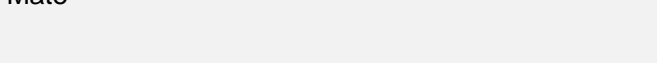 & $=0>1$ lt $/$ día & 50 & 39 & 7.9 & 2.7 & 1.3 & 5.7 & 0.0049 \\
\hline Dientes perdidos & $=0<13^{*}$ & 52 & 15 & 79 & 27 & 12 & 56 & 0005 \\
\hline & $>13$ & 48 & 38 & 1.0 & 2.1 & 1.0 & 0.0 & \\
\hline Consumo acumulado de tabaco (cigarrillos) & $<100000^{*}$ & 79 & 32 & 603 & 0 & 11 & 51 & 001 \\
\hline & $1000000+$ & 21 & 21 & & & 1.1 & & 0.017 \\
\hline Sexo & Femenino* & 64 & 24 & & & & & \\
\hline & Masculino & 36 & 29 & 4.0 & 2.1 & 1.1 & $\mathbf{T} . \mathbf{2}$ & 0.0220 \\
\hline Candidiasis crónica & $\mathrm{No}^{*}$ & 89 & 43 & & 10 & 07 & 16 & 017 \\
\hline & Sí & 11 & 10 & & & & & \\
\hline Exposición a carcinógenos ambientales y laborales ${ }^{\circ}$ & $<10^{*}$ & 82 & 40 & & & & & \\
\hline & $100+$ & 18 & 13 & 0.01 & 1.4 & 0.0 & 0.0 & 0.04 \\
\hline Tabaquismo pasivo ${ }^{\circ}$ & $=0<20^{*}$ & 61 & 28 & 005 & 11 & 07 & ר & 000 \\
\hline & $>20$ & 39 & 25 & 0.0 & 1.4 & 0.1 & 2.1 & 0.00 \\
\hline Dieta protectora (porc/sem) & $160-$ & 50 & 28 & 01 & 08 & 01 & 17 & 074 \\
\hline & $>16$ & 50 & 25 & 0.1 & 0.0 & 0.4 & 1.1 & 0.17 \\
\hline Historia familiar de cáncer & $\mathrm{No}^{*}$ & 35 & 23 & & & & & \\
\hline & Sí & 65 & 30 & 1.04 & 0.1 & 0.0 & 1.4 & 0.00 \\
\hline $\begin{array}{l}{ }^{\circ} \text { en años } \\
{ }^{*} \text { Categoría de referencia }\end{array}$ & & & & & & & & \\
\hline
\end{tabular}


Tabla 2: Regresión logística ajustada por edad, género, tabaco y alcohol

\begin{tabular}{|l|c|c|c|}
\hline Variable & OR (IC 95\%) & X' Wald & $p$-valor \\
\hline $\begin{array}{l}\text { Desórdenes potencialmente } \\
\text { malignos }\end{array}$ & $39,94(9,14-174,5))$ & 24,01 & $\mathbf{0 , 0 0 0 1}$ \\
\hline VPH & $15,78(3,88-64,24)$ & $14,84,0001$ & $\mathbf{0 , 0 0 1 2}$ \\
\hline $\begin{array}{l}\text { Consumo de agua de pozo en } \\
\text { zona arsenical }\end{array}$ & $8,21(2,3-29,3)$ & 8,51 & $\mathbf{0 , 0 0 3 5}$ \\
\hline Bajo consumo de mate & $3,26(1,47-7,21)$ & 13,94 & $\mathbf{0 , 0 0 0 2}$ \\
\hline Irritación mecánica crónica & $4,84(2,12-11,08)$ & 1,39 & 0,23 \\
\hline Dientes perdidos & $1,62(0,73-3,59)$ & & \\
\hline Todas las variables fueron analizadas de manera separada y ajustadas por edad, género, tabaco y alcohol
\end{tabular}

\begin{tabular}{|l|c|c|c|}
\hline \multicolumn{1}{|c|}{ Vabla 3: Regresión logística, modelo final } & OR (IC 95\%) & X² Wald & p-valor \\
\hline $\begin{array}{l}\text { Consumo de agua de pozo en } \\
\text { zona arsenical }\end{array}$ & $25,75(4,74-139,9)$ & 14,16 & $\mathbf{0 , 0 0 0 2}$ \\
\hline $\begin{array}{l}\text { Desórdenes potencialmente } \\
\text { malignos }\end{array}$ & $24,65(4,38-138,8)$ & 13,21 & $\mathbf{0 , 0 0 0 3}$ \\
\hline VPH & $10,42(1,91-56,8)$ & $\mathbf{0 , 0 0 6 8}$ \\
\hline Irritación mecánica crónica & $6,28(1,75-22,5)$ & $\mathbf{0 , 0 0 4 9}$ \\
\hline Bajo consumo de mate & $4,80(1,28-17,9)$ & 5,42 & $\mathbf{0 , 0 1 9 9}$ \\
\hline Edad mayor a 45 años & $4,27(1,01-18,0)$ & 3,91 & $\mathbf{0 , 0 4 8}$ \\
\hline Consumo pesado de Tabaco & $3,61(1,00-12,9)$ & 3,84 & $\mathbf{0 , 0 5}$ \\
\hline Consumo pesado de Alcohol & $3,24(0,70-14,9)$ & 2,27 & 0,1316 \\
\hline Sexo & $1,64(0,39-6,8)$ & 0,46 & 0,4968 \\
\hline
\end{tabular}




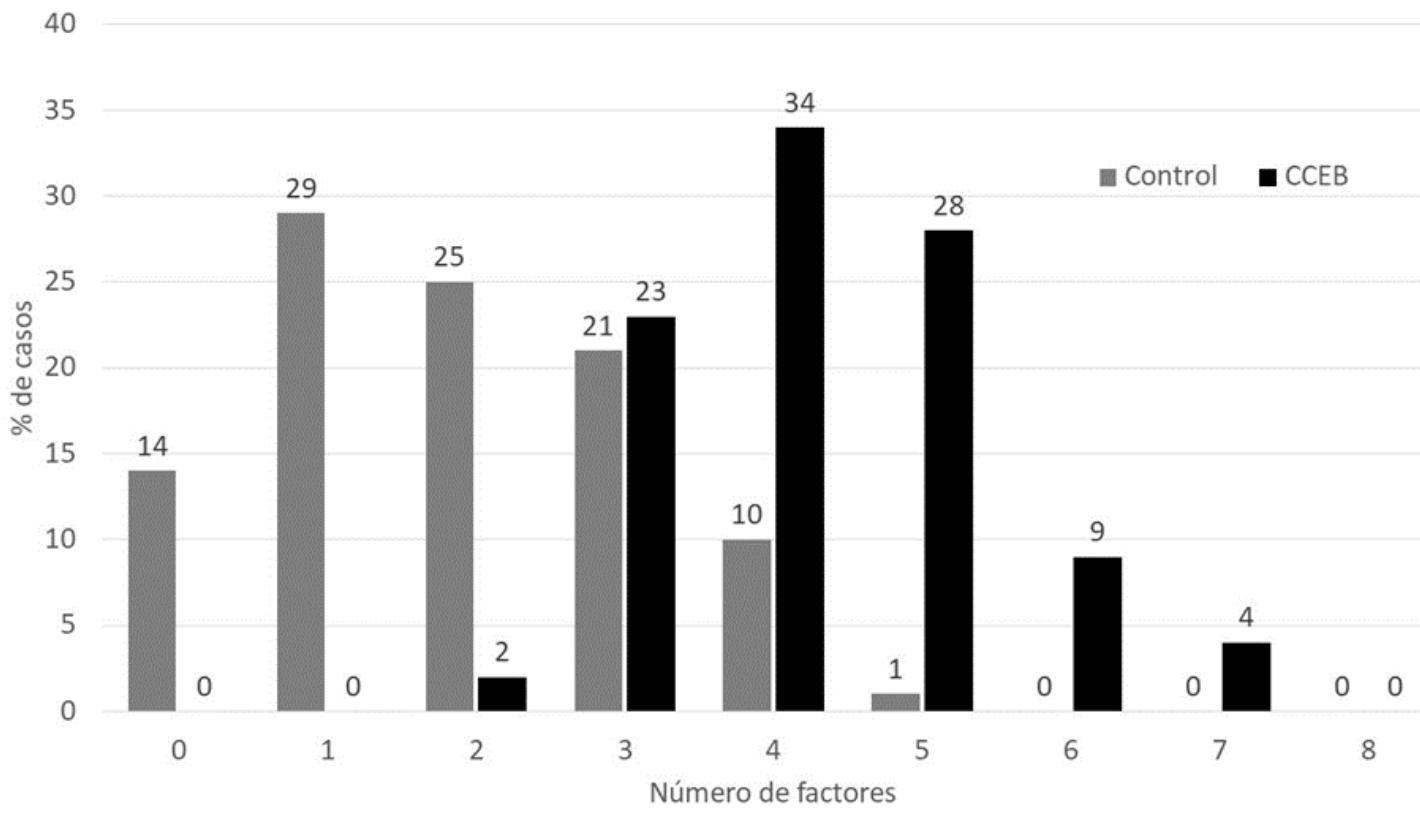

Figura 1: Distribución de pacientes según número de factores de riesgo

\begin{tabular}{|c|c|c|c|c|c|}
\hline Número de factores & $\begin{array}{c}\text { Control } \\
n=100(\%)\end{array}$ & $\begin{array}{c}\text { CCEB } \\
n=53(\%)\end{array}$ & $x^{2}$ & OR (IC $95 \%)$ & $p$-valor \\
\hline 0 & $14(14)$ & $0(0)$ & \multirow{9}{*}{82,49} & & \multirow{9}{*}{$\begin{array}{c}< \\
0.0001\end{array}$} \\
\hline 1 & $29(29)$ & $0(0)$ & & & \\
\hline 2 & $25(25)$ & $1(2)$ & & & \\
\hline 3 & $21(21)$ & $12(23)$ & & & \\
\hline 4 & $10(10)$ & $18(34)$ & & & \\
\hline 5 & $1(1)$ & $15(28)$ & & & \\
\hline 6 & $0(0)$ & $5(9)$ & & & \\
\hline 7 & $0(0)$ & $2(4)$ & & & \\
\hline 8 & $0(0)$ & $0(0)$ & & & \\
\hline 0 a 2 & $68(68)$ & $1(2)$ & \multirow{3}{*}{77,08} & & \multirow{3}{*}{$\begin{array}{c}< \\
0.0001\end{array}$} \\
\hline 3 a 4 & $31(31)$ & $30(56,5)$ & & & \\
\hline 5 o más & $1(1)$ & $22(41,5)$ & & & \\
\hline 0 a 2 & $68(68)$ & $1(2)$ & \multirow{2}{*}{61,15} & \multirow{2}{*}{$110,5(20,6-590)$} & \multirow{2}{*}{$\underset{0.0001}{<}$} \\
\hline 3 o más & $32(32)$ & $52(98)$ & & & \\
\hline
\end{tabular}




\section{Discusión}

Las poblaciones humanas y los sistemas ecológicos están comúnmente expuestos a una mezcla diversa y dinámica de factores estresantes biológicos, químicos, físicos y psicosociales como parte de su existencia diaria. Cuando las cuestiones que debe abordar la evaluación de riesgos implican evaluar las probabilidades conjuntas de daño de una serie de factores estresantes, exigen los enfoques conceptuales y los métodos de evaluación de la evaluación del riesgo acumulativo $^{(11)}$. El concepto de CCEB como enfermedad multifactorial requiere aplicar estos criterios de riesgo acumulativo, iniciando el análisis a partir de un mecanismo simple como la sumatoria de los factores de riesgo.

Independientemente de algunas consideraciones sobre ciertos factores de riesgo, el presente estudio mostró que estar o haber estado expuesto a mayor número de factores de riesgo de CCEB genera a su vez un riesgo mucho mayor que cada factor individual. Esto fue evidente más allá del test o la categorización que se utilizaron para analizar esta relación, a punto tal que tener 3 o más factores de riesgo generó un aumento del riesgo con un OR de más de 110, muy superior a cada factor incluido en el estudio.

A su vez, el área bajo curva ROC con el que validó el resultado de la categorización según número de factores, con valor mayor a 0,9, sugiere que a partir de estos resultados podría desarrollarse un sistema con sensibilidad y especificidad suficientes como para emplearlo en la detección de pacientes de alto riesgo de CCEB.

De acuerdo con la categorización del número de factores, la interpretación de los resultados puede presentar varios matices. La primera categorización analizada, considerando categorías de 0 a 8 factores, mostró que a medida que se incrementa el número de factores, aumenta la presencia de CCEB. En las categorías de 0 a 2 factores, como prácticamente no hubo CCEB, podrían ser consideradas de bajo riesgo. Entre los pacientes que presentaron 3 o 4 factores, el porcentaje de pacientes con cáncer bucal fue sustancialmente mayor que los que tuvieron 2 factores o menos, por lo que podrían considerarse de alto riesgo. $Y$ en los pacientes que presentaron 50 más factores, prácticamente no hubo pacientes del grupo control, por lo que podrían ser considerados de muy alto riesgo. Y si se pretende simplificar aún más la interpretación, tener 3 o más factores de riesgo generó un alto riesgo de CCEB. Todos estos criterios podrían ser útiles para establecer pauta de prevención y/o información para los pacientes, destacando que, por ejemplo, el sólo hecho de fumar no determina qué paciente va a desarrollar CCEB, sino que deben coexistir otros factores. Esto no resta importancia o gravedad al hábito de fumar, sino que explica al menos en parte, por qué no todos los fumadores desarrollan cáncer bucal, incluso los fumadores pesados. Criterios similares podría aplicarse a cada uno de los factores, ya que ninguno de los factores analizados como tampoco ninguna combinación de factores, estuvieron presentes en la totalidad de los casos, y por lo tanto ninguno puede explicar la totalidad de los CCEB.
El mayor riesgo de CCEB asociado al mayor número de factores no debiera explicarse sólo por una cuestión matemática. Los diferentes factores de riesgo pueden presentar múltiples interacciones específicas. Es conocida la interacción entre tabaco y alcohol, pero en principio, cada factor de riesgo podría interactuar con los otros. Por ejemplo, el tabaco posee un efecto carcinogénico fundamentalmente iniciador; pero tanto en fumadores como ex fumadores estos daños podrían ser agravados por el efecto carcinogénico promotor de la inflamación asociada a IMC ${ }^{(12)}$.

El análisis de los factores de riesgo de CCEB se realiza generalmente a través de modelos multivariados de regresión logística, que nos indican cuáles factores están asociados a CCEB habiendo ajustado según otras variables. Esta metodología no nos permite analizar la interacción de factores de riesgo, para lo cual es conveniente recurrir a otras estrategias, como estratificación según exposición a las variables de riesgo, o reglas de predicción clínica. Un ejemplo de lo primero se puede observarse en el efecto de la interacción de tabaco, alcohol y masticación de betel, que muestras efectos aditivos o multiplicativos en el riesgo de CCEB según las diferentes categorías de interacción de dichas variables $\left.{ }^{(13}\right)$. La segunda estrategia puede observarse en estudios que analizan el riesgo de desarrollar desórdenes orales potencialmente malignos (DOPM), precursores del CCEB, en el que la ponderación y sumatoria de los odds ratio de los factores incluidos, permite detectar con adecuada sensibilidad y especificidad qué pacientes presentan mayor riesgo de padecer $\mathrm{DOPM}^{(14)}$. Si bien ambas estrategias son correctas y muestran la interacción de factores de riesgo, no mencionan expresamente lo que el presente estudio muestra, en referencia a que la acumulación de factores de riesgo podría ser más importante que cualquier factor considerado individualmente, o incluso que cualquier interacción específica.

En cuanto a algunos factores individuales, es necesario abordar algunos aspectos contrastantes con los conocimientos existentes sobre los mismos. El primero de ellos es que tanto el tabaco como el alcohol no fueron los factores de riesgo más potentes entre los analizados. Esto no significa que estos factores no sean verdaderas causas de cáncer bucal, sino que simplemente evidencian que en los pacientes con CCEB incorporados al presente trabajo la exposición a eso factores fue baja, probablemente por disminución en los hábitos de consumo de tabaco y de alcohol como también por el elevado consumo existente en el grupo control. Es necesario resaltar la prevalencia de factores de riesgo de CCEB presenta una variación geográfica importante ${ }^{(15)}$, por lo que estos resultados deben considerarse con cuidado si se pretende extrapolarlos a otras poblaciones. Estos criterios pueden ser también aplicados a todos los factores que no fueron encontrados con asociación estadísticamente significativa con mayor riesgo de CCEB. Otro resultado controversial es el consumo de mate como factor de riesgo de cáncer bucal. Los antecedentes muestran que el mayor consumo de mate genera un mayor riesgo de CCEB, principalmente por el efecto de la alta temperatura del agua ${ }^{(16)}$ y quizás también por la presencia de agroquímicos contaminantes, que superarían los efectos antioxidantes naturales de la yerba mate (Ilex paraguariensis). 
Aun así, en nuestra población estudiada, el CCEB estuvo asociado con un menor consumo de mate, no con un mayor consumo. La posible explicación de este fenómeno es que en nuestra población el consumo diario de mate tuvo un promedio apenas superior a 1 litro por día, mientras que en los estudios que sostienen la relación de CCEB con mayor consumo de mate el promedio diario fue casi el doble. Estos hallazgos podrían plantear que, en bajas dosis de consumo de mate, prevalecen los efectos protectores del mismo, mientras que, superado el límite de un litro diario, prevalece el efecto de irritación térmica del agua caliente.

Por último, es importante destacar que, entre los cuatro factores de riesgo más potentes, 3 de ellos son de valoración clínica (DPM, VPH e IMC), lo que pone de manifiesto que según estos modelos sería muy difícil valorar el riesgo individual de cada paciente sin una inspección clínica de la mucosa bucal por un operador calibrado en el diagnóstico de dichas variables.

La exposición a múltiples factores de riesgo de CCEB es el principal factor de riesgo de CCEB, constituyéndose prácticamente en una causa

\section{Bibliografía}

1. Carbone M, Pass HI. Multistep and multifactorial carcinogenesis: when does a contributing factor become a carcinogen? Semin Cancer Biol. 2004;14:399-405.

2. Campo-Trapero J, Cano-Sánchez J, Palacios-Sánchez B, Sánchez-Gutierrez JJ, González-Moles MA, Bascones-Martínez A. Update on molecular pathology in oral cancer and precancer. Anticancer Res. 2008;28:1197-205.

3. Di Credico, G. et al. Joint effects of intensity and duration of cigarette smoking on the risk of head and neck cancer: $A$ bivariate spline model approach. Oral Oncol. 94, 47-57 (2019).

4. Warnakulasuriya S. Risk assessment in oral cancer. In: Chapple ILC, Papapanou PN, editors. Risk Assessment in Oral Health: A Concise Guide for Clinical Application. Springer International Publishing; 2020. p. 119-32.

5. Radoï L, Luce D. A review of risk factors for oral cavity cancer: the importance of a standardized case definition. Community Dent Oral Epidemiol. 2013;4:97-109, e78-91.

6. Warnakulasuriya S. Causes of oral cancer--an appraisal of controversies. Br Dent J. 2009;207:471-5.

7. Litter MI, Ingallinella AM, Olmos V, Savio M, Difeo G, Botto L, et al. Arsenic in Argentina: Occurrence, human health, legislation and determination. Sci Total Environ. 2019;676:756-66.

8. Deneo-Pellegrini $H$, De Stefani $E$, Boffetta $P$, Ronco $A L$, Acosta G, Correa $P$, et al. Maté consumption and risk of oral cancer: Case-control study in Uruguay. Head Neck. 2013;35:1091-5.

9. Piemonte E, Lazos J, Belardinelli P, Secchi D, Brunotto M, Lanfranchi-Tizeira $\mathrm{H}$. Oral cancer associated with chronic mechanical irritation of the oral mucosa. Med Oral Patol Oral Cirugia Bucal. 2018;23:e151-60.

10. Warnakulasuriya S. Oral potentially malignant disorders: $A$ comprehensive review on clinical aspects and management. Oral Oncol. 2020;102:104550.

11. Callahan, M. A. \& Sexton, K. If Cumulative Risk Assessment Is the Answer, What Is the Question? Environ. Health Perspect. 115, 799-806 (2007).

12. Pérez, M. A., Raimondi, A. R. \& Itoiz, M. E. An experimental model to demonstrate the carcinogenic action of oral chronic traumatic ulcer. J. Oral Pathol. Med. Off. Publ. Int. Assoc. Oral Pathol. Am. Acad. Oral Pathol. 34, 17-22 (2005). necesaria de CCEB. Por ello, un índice de riesgo multifactorial, derivado de estos resultados, podría ser una herramienta sensible y específica para la detección de individuos con alto riesgo de cáncer bucal.

\section{Limitaciones de responsabilidad}

La responsabilidad del trabajo es exclusivamente de los autores.

\section{Conflictos de interés}

Ninguno

Originalidad del trabajo

Este artículo es original y no ha sido enviado para su publicación a otro medio de difusión científica en forma completa ni parcialmente.

\section{Cesión de derechos}

Los participantes de este trabajo ceden el derecho de autor a la Universidad Nacional de Córdoba para publicar en la Revista de la Facultad de Ciencias Médicas y realizar las traducciones necesarias al idioma inglés.

\section{Participación de los autores}

Todos los autores han participado en la concepción del diseño, recolección de la información y elaboración del manuscrito, haciéndose públicamente responsables de su contenido y aprobando su versión final.

13. Petti, S., Masood, M. \& Scully, C. The magnitude of tobacco smoking-betel quid chewing-alcohol drinking interaction effect on oral cancer in South-East Asia. A meta-analysis of observational studies. PloS One 8, e78999 (2013).

14. Amarasinghe, H. K., Johnson, N. W., Lalloo, R., Kumaraarachchi, M. \& Warnakulasuriya, S. Derivation and validation of a risk-factor model for detection of oral potentially malignant disorders in populations with high prevalence. Br. J. Cancer 103, 303-309 (2010). 15. Warnakulasuriya S. Global epidemiology of oral and oropharyngeal cancer. Oral Oncol. 2009;45:309-16.

16. Mello FW, Scotti FM, Melo G, Warnakulasuriya S, Guerra ENS, Rivero ERC. Maté consumption association with upper aerodigestive tract cancers: A systematic review and meta-analysis. Oral Oncol. 2018:82:37-47. 